\title{
Lower Serum Phosphate Ion Level is Associated with Acute Hydrocephalus After Aneurysmal Subarachnoid Hemorrhage.
}

\section{De-Zhi Kang ( $\sim$ kdz99988@vip.sina.com )}

First Affiliated Hospital of Fujian Medical University

\section{Yi-Bin Zhang}

First Affiliated Hospital of Fujian Medical University

\section{Shu-Fa Zheng}

First Affiliated Hospital of Fujian Medical University

\section{Guo-Rong Chen}

First Affiliated Hospital of Fujian Medical University

\section{Chun-Wang Li}

First Affiliated Hospital of Fujian Medical University

\section{Yuan-Xiang Lin}

First Affiliated Hospital of Fujian Medical University

\section{Pei-Sen Yao}

First Affiliated Hospital of Fujian Medical University

\section{Research}

Keywords: Subarachnoid hemorrhage, Aneurysm, Hydrocephalus, Risk factor, Phosphate

Posted Date: August 23rd, 2021

DOl: https://doi.org/10.21203/rs.3.rs-806098/v1

License: (c) (i) This work is licensed under a Creative Commons Attribution 4.0 International License. Read Full License 


\section{Abstract}

Background: The relationship between serum phosphate ion (sPi) and the occurrence of acute hydrocephalus (aHCP) in aneurysmal subarachnoid hemorrhage (aSAH) remains largely unknown and controversial. The primary aim of this study was to evaluate the association between sPi on admission and aHCP following aSAH.

Methods: A prospective study was conducted on six hundred thirty-five patients over the age of 19 years diagnosed with aSAH in our institution from January 21, 2012, to December 30, 2019. Data on clinical characteristics, laboratory parameters, treatments, and outcomes were collected and analyzed. The association between lower SPi levels and aHCP was assessed in univariate and multivariate analyses. Propensity-score matching (PSM) analyses were adopted to reduce the baseline differences between the non-HCP group and HCP group.

Results: The overall incidence of aHCP following aSAH was 19.37\% (123/512). Lower sPi levels were detected in patients with aHCP compared with those without [0.86(0.67-1.06) $\mathrm{mmol} / \mathrm{L}$ vs. 1.04(0.84-1.21)] $\mathrm{mmol} / \mathrm{L}$ in the univariate analysis. In the multivariate analysis, lower sPi, high modified Fisher (mFisher) grade, and high Hunt-Hess grade were associated with aHCP [odds ratios (OR) 1.729, 95\% Cl 1.1392.623, $\mathrm{P}=0.01$; mFisher OR 0.097,95\% Cl 0.055-0.172, $\mathrm{p}<0.001$; Hunt-Hess, OR 0.555, $95 \%$ confidence interval $(\mathrm{Cl}) 0.320-0.961, \mathrm{P}=0.036$ ]. After PSM, the matched HCP group had a significantly lower sPi level than the matched non-hydrocephalus group $[(0.86(0.67-1.06)$ vs. $0.94(0.76-1.12) \mathrm{mmol} / \mathrm{L}, \mathrm{P}=0.020)]$. The area under the curve (AUC) of the sPi level and the logistic regression model based on these predictors (sPi, Hunt-Hess grade, and mFisher score) was 0.667 and 0.840 (sensitivity of $88.6 \%$ and specificity of 68.4\%) for predicting aHCP, respectively.

Conclusion: Lower sPi levels at admission were observed in patients with unfavorable outcomes. Lower sPi levels were associated with aHCP and poor prognosis, and the model constructed by sPi levels, HuntHess grade, and mFisher grade significantly improves the prediction of aHCP after aSAH.

\section{Background}

Aneurysmal subarachnoid hemorrhage is a fast developing, devastating, and life-threatening hemorrhagic stroke with high mortality and disability[1-3]. Hydrocephalus (HCP) is a common complication after aSAH, with a reported incidence ranging from $6.5 \%$ to $85 \%[4-7]$, which is classified as acute $(0-3$ days post-SAH), subacute ( $4-13$ days post-SAH), or chronic ( 14 days post-SAH) $[8,9]$. Acute hydrocephalus (aHCP) is a potentially treatable cause of early neurological deterioration, and $20 \%$ of aSAH patients develop aHCP within 72 hours $[6,8]$.

Numerous factors have been reported in the literature to predict the occurrence of aHCP following aSAH, including female sex, elderly age, higher Fisher grade on initial CT, presence of intraventricular hemorrhage, rebleeding, and laboratory findings $[1,7,8,10,11]$. Many efforts have been made in studying sensitive biomarkers for predicting aHCP[12], but reports of risk factors for its development are 
inconsistent[13]. Clinically, laboratory biomarkers have received extensive attention and research due to their convenience, practicality, and sensitivity[14]. Nonetheless, most basic research biomarkers require special laboratory tests or cerebrospinal fluid (CSF) sample collection, which may impose additional risk and expense[14]. Therefore, it is necessary and valuable to explore convenient, sensitive, and inexpensive biomarkers for the clinical practice of aSAH treatment.

In the early stages of aHCP, the systemic and complex inflammatory response is crucial in the pathophysiological process of the formation of aHCP and is closely related to the increased risk of a worse prognosis[12]. Identifying high-risk aHCP patients in the early stage of aSAH is sufficiently important to provide timely appropriate interventions. The feasibility of using biomarkers to identify these high-risk patients with aHCP remains challenging[12]. Phosphate participates in many physiological processes in the human body, such as the composition of enzymes, and is regulated by multiple organs such as the kidneys, bones, and digestive system $[15,16]$. However, in acute disease, the body's phosphate metabolism cannot be regulated accurately[15]. Various studies have shown that sPi levels are closely related to the occurrence and prognosis of acute cerebrovascular disease[17-20]. Hypophosphatemia is a common treatable problem in the intensive care unit (ICU) and manifests many pathophysiological processes that occur in critical illness, including hemorrhagic stroke and other neurocritical diseases[21, 22]. Weber et al. reported for the first time that profound changes in serum phosphate might be related to reversible brain lesions[18]. Subsequently, impressive literature reported that hypophosphatemia is associated with a disturbance of consciousness after spontaneous intracerebral hemorrhage (sICH), but its mechanism is still poorly understood[19]. Emerging literature indicated that hypophosphatemia is associated with HCP after sICH in the ICU[17]. However, the patients enrolled in this study were mixed with other causes of intracerebral hemorrhage, and the number of patients with $\mathrm{ICH}$ was small, so there was a particular research bias.

To the best of our understanding, no previous studies on aSAH have evaluated the effect of sPi level on aHCP after aSAH. We sought to investigate whether sPi level was linked to the severity of aSAH or other factors, such as sex, age, and blood pressure. Therefore, this study was performed to determine whether sPi level was associated with aHCP in aSAH patients.

\section{Methods}

\section{Study Population}

This was a single-center, prospective observational study of aSAH patients admitted to the neurosurgery department in the First Affiliated Hospital of Fujian Medical University between January 21, 2012, and December 30, 2019. The study protocol was reviewed and approved by the hospital's institutional review board. The patients or their relatives gave informed consent. Patients were included in the analysis with the following inclusion criteria: 1) age $\geq 19$ years old; 2 ) Computed tomography scans confirmed the diagnosis of SAH on admission; 3) Computed tomography angiography (CTA) and/or digital subtraction angiography (DSA) diagnosed subarachnoid hemorrhage due to a ruptured cerebral aneurysm; 4) survival 
for no less than 72 hours after aSAH to allow assessment of the development and prognosis of hydrocephalus. While exclusion criteria were listed as following: 1) age of $<19$ years; 2 ) a history of neurological disease including cerebrovascular disease, hemorrhagic or ischemic stroke and trauma;3) patients presented with postoperative hydrocephalus;4) a history of familial hypophosphatemia or parathyroid disease;5) patients underwent emergency aneurysm clipping or interventional embolization within 72 hours after aSAH;6)acute kidney injury or chronic kidney disease;7)concurrent systemic comorbidities. The flowchart of this study is present in Figure 1.

Clinical management followed the American Heart Association and American Stroke Association guidelines[7].

\section{Baseline data collection}

We collected peripheral venous blood samples within 30min of admission. The reference range of the serum phosphorus level in our institutions was $0.85-1.51 \mathrm{mmol} / \mathrm{L}$. After admission, the clinical data and demographic information were obtained from the institutional electronic medical record database, including age, sex, medical history, time from onset to admission, Hunt-Hess grade, mFisher score, aneurysm location, and laboratory data. aSAH patients were grouped into mild aSAH (mFisher score 1-2) or severe aSAH (mFisher score 3-4) according to CT on admission and were also grouped into mild

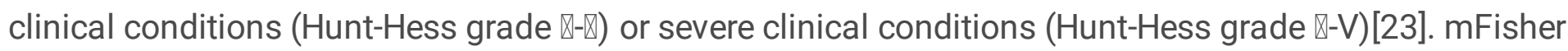
score was dichotomized as "low grade (Grade 0,1, 2)" and "high grade (Grade3, 4)", Hunt-Hess grade as

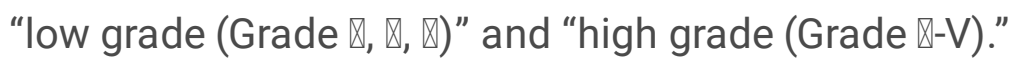

\section{Diagnosis and treatment of hydrocephalus}

The onset of hydrocephalus is rapid and persistent, occurring within 6 hours after aSAH and remaining for at least 72 hours. Therefore, aHCP was defined as the development of ventricle enlargement within 72 $\mathrm{h}$ of the aneurysmal rupture. The bicaudate index and relative bicaudate index were used to diagnose hydrocephalus[10]. Two neurosurgeons and two radiologists independently evaluated the diagnostic imaging findings of acute hydrocephalus, and the consensus made the final judgment of more than three people. A following-up CT was performed depending on the patient's clinical condition. Emergency brain CT scans were performed in the presence of clinical deterioration, new focal neurological deficits, seizures, or gradual loss of consciousness, including rebleeding or aHCP. If the patient had severe acute hydrocephalus or progressive deterioration of consciousness or periventricular edema, immediate external ventricular drainage was performed[3].

\section{Outcome Assessment}


All patients were followed up until 90 days after onset of aSAH or until death, depending on which happened first. Functional outcome was assessed 90 days after the aSAH onset using the modified Rankin scale (mRS) through follow-up, which was dichotomized into a favorable prognosis (mRS $₫ 3$ ) and an unfavorable (mRS 4-6) prognosis[24]. Follow-up information was gathered by outpatient reviews, telephone, or WeChat interviews. mRS scores range from 0 to 6 , with 0 indicating no symptoms, 1 no clinically significant disability, 2 slight disability, 3 moderate disability, 4 moderately severe disability, 5 severe disability, and 6 death[25]. Clinical outcome was evaluated by trained research coordinators who were blinded to the subject's identity or clinical information.

\section{Statistical Analysis}

All statistical analyses were conducted by SPSS software (version 25.0, IBM SPSS, IBM Corp), Prism 8.3.0 (GraphPad Software, San Diego, CA, USA), and MedCalc version 20.0.4 (MedCalc Software, Ostend, Belgium). For comparison of different groups for categorical variables, the $\chi 2$ test or Fisher's exact test was utilized. Normally distributed continuous variables are presented as means \pm standard deviation (SD) employing Student t-test, and non-normally distributed variables as median (interquartile range, IQR) using the Mann-Whitney $U$ test or Kruskal-Wallis test intergroup differences. Median with IQR is shown for all scatter plots. Spearman's correlation analysis was performed to evaluate the correlation coefficient between two variables. All Clinical variables associated with hydrocephalus at $P<0.10$ in the univariate analysis were considered for entry into the multivariate models. In the multivariate model, sPi was dichotomized as " $\leq$ optimal cutoff value" and"> optimal cutoff value." Multivariable logistic regression models were used to develop the combined models. The receiver operating characteristic (ROC) curve was constructed, and the area under the AUC was calculated to evaluate the predictive power of the sPi for aHCP. Also, a combined model (constructed by multivariable logistic regression) ROC analysis was created to potentially identify a more potent predictive hydrocephalus model. PSM analysis was conducted to further account for significant differences in baseline characteristics between HCP and nonHCP groups. Covariates associated with hydrocephalus were included in the matching. The nearest neighbor matching algorithm was performed to match the HCP group and the non-CP group at a ratio of 1:1. $P<0.05$ was accepted as statistically significant.

\section{Results}

\section{Patient characteristics}

Six hundred thirty-five aSAH patients were enrolled in the study based on the inclusion criteria between January 21, 2012, and December 30, 2019. The mean age was (54.26 \pm 10.77$)$ years, and four hundred (63\%) participants were female. Ninety-six patients (15.1\%) were in severe clinical condition on admission (Hunt-Hess grade $\mathbb{\Downarrow}-\mathrm{V}$ ). Two hundred fifty-nine patients $(40.8 \%$ ) had severe aSAH (mFisher score 3-4) on admission CT. The concentrations of serum potassium, sodium, calcium and SPi at hospital admission were 3.94(3.65-4.22) mmol/L, 140.80(138.0-143.0) $\mathrm{mmol} / \mathrm{L}, 2.17(2.08-2.26) \mathrm{mmol} / \mathrm{L}$, 
$1.01(0.82-1.19) \mathrm{mmol} / \mathrm{L}$, respectively. Aneurysms were treated with surgical clipping in $436(68.7 \%)$ patients, while the other 199 (31.3\%) received endovascular coiling. All enrolled participants were segregated into the non-HCP group $(n=512)$ and HCP group $(n=123)$. The overall incidence of aHCP following aSAH was $19.37 \%(123 / 512)$.

\section{Association of sPi Levels with aHCP}

Demographic characteristics, clinical features, and laboratory data with potential significance for aHCP are summarized in Table1. On univariate analysis, factors associated with the occurrence of aHCP following aSAH were hypertension $(P<0.001)$, diabetes mellitus $(P=0.007)$, systolic blood pressure $(S B P)$ $(P<0.001)$, diastolic blood pressure (DBP) $(P=0.021)$, admission Hunt-Hess grade $(P<0.001)$, mFisher scale $(P<0.001)$, serum potassium $(P=0.004)$, serum calcium $(P=0.042)$ and $s P i(P<0.001)$. Lower sPi levels were detected in patients with aHCP compared with those without $[0.86(0.67-1.06) \mathrm{mmol} / \mathrm{L}$ vs. 1.04(0.841.21) $\mathrm{mmol} / \mathrm{L}, \mathrm{P}<0.001]$. (Table1 and Fig.2A). Multivariable analysis revealed that lower sPi level, higher mFisher score, and higher Hunt-Hess grade were associated with aHCP (Table 1; sPi, OR $1.729,95 \% \mathrm{Cl}$ 1.139-2.623, $\mathrm{P}=0.01$; $\mathrm{mFisher}$ OR 0.097,95\% Cl 0.055-0.172, $\mathrm{P}<0.001$; Hunt-Hess, OR 0.555, $95 \% \mathrm{Cl}$ $0.320-0.961, P=0.036$ ), while hypertension, diabetes mellitus, $S B P, D B P$, serum potassium and serum calcium were not associated with aHCP.

\section{Table 1}

\section{Univariate and multivariate analyses of association with hydrocephalus following aSAH}

After PSM, no significant differences were observed in hypertension, diabetes mellitus, SBP, DBP, serum potassium and serum calcium, admission Hunt-Hess grade, Fisher grade between HCP and non-HCP groups. The matched HCP group had a significantly lower sPi level than the matched non-HCP $[(0.86(0.67-1.06)$ vs. 0.94(0.76-1.12)) $\mathrm{mmol} / \mathrm{L}, \mathrm{P}=0.020$; Fig. $2 \mathrm{~B}]$.

ROC curve analysis shows an AUC (area under the curve) of 0.667 (95\% Cl 0.629-0.704, Pख0.0001) (Sensitivity=63.4\%; Specificity=66.8\%), and the best cutoff value for sPi level as a predictor for aHCP in aSAH patients was determined as $0.93 \mathrm{mmol} / \mathrm{L}$. The AUC of the logistic regression model based on these predictors (sPi, Hunt-Hess grade, and mFisher grade) was 0.840 (95\% $\mathrm{Cl} 0.809-0.868, \mathrm{P} \unrhd 0.0001)$ with a sensitivity of $88.6 \%$ and specificity of $68.4 \%$ for aHCP, which is a stronger hydrocephalus predictor than a

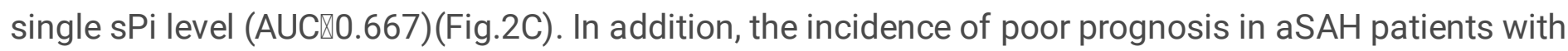
HCP was higher than those without HCP (Fig. 2D).

\section{Association of sPi level with clinical and radiologic status on admission}

The sPi level was significantly lower in aSAH patients with mFisher grade (3-4) compared to mFisher (1- 
Univariate

Characteristics

Non-

Hydrocephalus

Hydrocephalus

$$
(n=123)
$$$$
(n=512)
$$

Age, mean (SD) y

Gender, female (N,

$\%)$

\section{Medical history}

Smoking $(\mathrm{N}, \%)$

Alcohol (N, \%)

Hypertension ( $N, \%) \quad$ 288(56.3)

45(8.8)

$(\mathrm{N}, \%)$

Hyperlipidemia ( $\mathrm{N}$,

$\%)$

Admission vital

signs

$\mathrm{SBP}, \mathrm{mmHg}$

DBP, $\mathrm{mmHg}$

Hunt-Hess grade $(\mathrm{N}$,

$\%)$

Grade $\mathbb{X}-\mathbb{Z}$

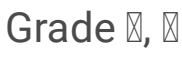

mFisher grade $(\mathrm{N}$,

\%)

Grade 0-2

Grade 3-4

Aneurysm characteristics $(\mathrm{N}$,

$\%)$

Multiple aneurysms

24(19.5)

44(35.8)

\begin{tabular}{ll}
$288(56.3)$ & $17(13.8)$ \\
\hline $224(43.8)$ & $106(86.2)$
\end{tabular}

$83.74 \pm 13.77$

$139.99 \pm 24.40$

$150.14 \pm 27.62$

$87.15 \pm 14.80$

0.021

$\bigotimes 0.001$

460(89.8)

$52(10.2)$

$<0.001$

17(13.8)

106(86.2)

$\llbracket 0.001$

79(64.2)
Multivariate analysis

OR $\quad 95 \% \mathrm{Cl} \quad P$ value

\section{$1.007 \quad 0.984-0.560$ 1.031}

\subsection{5}

0.282

0.518

$\varangle 0.001$

0.838

$0.489-\quad 0.518$

1.435

0.757

$0.390-\quad 0.412$

1.472

0.410

1.005

$0.992-\quad 0.445$

1.017

$0.974-\quad 0.672$ 1.017

$\begin{array}{lll}0.995 & 0.974- & 0.672 \\ & 1.017 & \end{array}$

$0.320-\quad 0.036$ 0.961

$\begin{array}{lll}0.555 & 0.320- & 0.036 \\ & 0.961 & \end{array}$

0.172

$\begin{array}{lll}0.097 & 0.055- & <0.001 \\ & 0.172\end{array}$


Anterior circulation $\quad$ 422(86.3) $\quad$ 108(87.8)

aneurysm

Time from onset to $\quad 14(10-17) \quad 15(6-17) \quad 0.354$

admission, $\mathrm{h}$

Admission

laboratory

$\begin{array}{lllllll}\text { Serum potassium, } & 3.97(3.67-4.24) & 3.83(3.45-4.12) & 0.004 & 1.006 & 0.609- & 0.980 \\ \text { mmol/L } & & & & & 1.662 & \end{array}$

$\begin{array}{llll}\text { Serum sodium, } & 140.8(138.33- & 141.0(137.70- & 0.906 \\ \mathrm{mmol} / \mathrm{L} & 142.88) & 143) & \end{array}$

$\begin{array}{lllllll}\text { Serum calcium, } & 2.18(2.08-2.27) & 2.15(2.08-2.24) & 0.042 & 0.830 & \begin{array}{l}0.145- \\ \text { mmol/L }\end{array} & 0.835\end{array}$

$\begin{array}{lllllll}\mathrm{sPi}, \mathrm{mmol} / \mathrm{L} & 1.04(0.84-1.21) & 0.86(0.67-1.06) & <0.001 & 1.729 & 1.139- & 0.01\end{array}$

2.623

Treatment ( $\mathrm{N}, \%)$

0.156

Clipping

Coiling DBP: Diastolic blood pressure; IQR: Interquartile range; mFisher: modified Fisher; OR: Odds ratios; sPi: serum Phosphate ion; SD: Standard deviation; SBP: Systolic blood pressure.

2) $[(1.81(1.49-2.28))$ vs. 1.42(1.20-1.67), Pख0.0001; Fig. 3A]. Furthermore, patients with Hunt-Hess (खV) had lower sPi concentration than those with Hunt-Hess (囚- $\mathbb{Z})[(0.85(0.64-1.10))$ vs. 1.03(0.84-1.20), P 0.0001; Fig. 3B]. Spearman analyses revealed a clear negative correlation between sPi and mFisher score $(r=-0.2348, P<0.001$, Fig.3B), between $s P i$ and Hunt-Hess grade $(r=-0.2913, P<0.001$,

Fig.3D), respectively. Correlation analyses revealed a negative correlation between sPi and SBP $(r=-0.137$, $\mathrm{P}=0.001)$. No correlation was detected between sPi and age or $\operatorname{sex}(\mathrm{P} \otimes 0.05)$.

Comparing patients in terms of sPi concentration, aHCP rate, the incidence of poor prognosis, mFisher scale, Hunt-Hess grade, and SBP were significantly higher in patients with lower $\mathrm{SPi}((\mathrm{sPi} \leq 1.0 \mathrm{mmol} / \mathrm{L})$ concentration than those with higher sPi concentration(sPi $₫ 1.0 \mathrm{mmol} / \mathrm{L})$. Moreover, serum potassium, sodium, and calcium were lower than patients with higher sPi concentrations (Table 3).

\section{Association of sPi Levels with Unfavorable 90 Days Functional Outcome}

Compared with the favorable functional group, the sPi level at admission was markedly lower in the unfavorable functional group [0.92(0.66-1.13) vs. 1.02(0.83-1.20) $\mathrm{mmol} / \mathrm{L}, \mathrm{P}=0.0005$, Fig.4A]. Patients with a sPi level below $1.0 \mathrm{mmol} / \mathrm{L}$ (the median level of $\mathrm{sPi}$ in all patients) have a more unfavorable outcome than patients with a sPi level $>1.0 \mathrm{mmol} / \mathrm{L}$ (Fig. 4B). 
According to the ROC curve, the AUC of sPi was 0.602 ( $95 \% \mathrm{Cl} 0.563-0.640 ; \mathrm{P}=0.003$ )

(Sensitivity=25.58\%; Specificity=91.99\%) for mRS based on a cut-off value of $0.66 \mathrm{mmol} / \mathrm{L}$ (Fig.4C).

\section{Table 2}

\section{Univariate analyses of association with hydrocephalus following aSAH after PSM}

\section{Table 3}

\section{Patients' demographics and baseline characteristics by sPi concentration}

\section{Discussion}

In the present study, we applied multivariate logistic regression analysis combined with PSM to investigate the relationship between sPi levels and aHCP. The significant findings of this study were as follows: 1) A lower admission sPi level was considered as a risk factor of aHCP; 2) mFisher scale and Hunt-Hess grade were negatively correlated with sPi, respectively; 3 ) Patients with severe aSAH (higher mFisher score and Hunt-Hess grade) have significantly lower sPi levels; 4) The sPi level was negatively associated with SBP;5) The model constructed by sPi levels, Hunt-Hess grade, and mFisher scale significantly improves the prediction of aHCP after aSAH. Risk factors for aHCP have been reported, including mFisher score, Hunt-Hess grade in aSAH patients, which were balanced in this study. After PSM, no significant differences were observed in the mFisher score, Hunt-Hess grade between the two groups. The matched aHCP group had a lower sPi level compared with the matched non-bleeding group in the univariate analysis. The sPi level was considered as a risk factor of aHCP, which was confirmed after PSM analysis. The AUC of the combined model was 0.840 with a sensitivity of $88.6 \%$ and specificity of $68.4 \%$ for aHCP. The model constructed by sPi levels, Hunt-Hess grade, and mFisher scale produced the best ability to predict aHCP compared with a single sPi level or mFisher scale, which was a valuable factor for predicting the occurrence of aHCP following aSAH. Previously reported literature demonstrated that aHCP and diffuse cerebral edema are more common in patients with non-traumatic intracranial hemorrhage accompanied by decreased sPi levels[17]. Based on it, we listed aSAH from non-traumatic $\mathrm{ICH}$ to explore the relationship between decrease sPi levels and aHCP. The present study consolidated and expanded previous observations[17] and constructed an unexpected and novel combined model for predicting aHCP.

Phosphate metabolism is an evolving field of basic and clinical research. Phosphorus is essential for all living cells, accounting for about $1 \%$ of human body weight. The most phosphate $(85 \%)$ is found in bones and teeth, and the remaining $15 \%$ is distributed in body fluids and other cells[15, 16]. Phosphate as an energy source in the form of ATP is essential for cell metabolism and the normal development of bones. Phosphate is an essential mineral that participates in many physiological pathways and mineralization, the essential elements of membrane composition, such as the formation of various enzymes, the 
Characteristics

$$
\text { Age, mean (SD) y }
$$

Gender, female (N, \%)

\section{Medical history}

Smoking $(\mathrm{N}, \%)$

Alcohol (N, \%)

Hypertension ( $\mathrm{N}, \%$ )

Diabetes mellitus ( $\mathrm{N}, \%)$

Hyperlipidemia (N, \%)

Admission vital signs

SAP, $\mathrm{mmHg}$

DBP, $\mathrm{mmHg}$

Hunt-Hess grade ( $N$, \%)

Grade $\mathbb{X}-\mathbb{\square}$

Grade $\otimes, \nabla$

mFisher grade $(\mathrm{N}, \%)$

Grade 0-2

Grade 3-4

Aneurysm characteristics ( $N, \%$ )

Multiple aneurysms

Anterior circulation aneurysm

Time from onset to admission, h

\section{Admission laboratory}

Serum potassium, $\mathrm{mmol} / \mathrm{L}$

Serum sodium, $\mathrm{mmol} / \mathrm{L}$

Serum calcium, $\mathrm{mmol} / \mathrm{L}$

$\mathrm{sPi}, \mathrm{mmol} / \mathrm{L}$
Non-Hydrocephalus

$(\mathrm{n}=108)$

$55.83 \pm 11.24$

66(61.1)

17(15.7)

10(9.30)

48(44.4)

13(12.0)

19(17.6)

$149.09 \pm 28.01$

$85.13 \pm 14.14$

77(71.3)

31(28.7)

17(15.7)

91(84.3)

18(16.7)

90(83.3)

13(8-16.75)

3.85(3.61-4.15)

140.55(138.0-142.10)

2.15(2.05-2.25)

0.94(0.76-1.12)

Page $10 / 24$

Hydrocephalus

$p$ value

$(n=108)$

$55.27 \pm 9.72$

0.693

74(68.5)

0.254

15(13.9)

0.702

11(10.2)

0.818

45(41.7)

0.680

19(17.6)

0.250

21(19.4)

0.726

$148.92 \pm 27.45$

0.963

$86.68 \pm 15.34$

0.442

0.134

73(61.9)

35(38.1)

1.00

17(15.7)

91(84.3)

0.444

14(13.0)

94(87.0)

15(6-17)

0.987 
Clipping

Coiling
$78(72.2)$

$30(27.8)$
$80(74.1)$

28(25.9)

aSAH: aneurysmal subarachnoid hemorrhage; aHCP: acute hydrocephalus; $\mathrm{Cl}$ : Confidence interval; DBP: Diastolic blood pressure; IQR: Interquartile range; mFisher: modified Fisher; OR: Odds ratios; sPi: serum Phosphate ion; SD: Standard deviation; SBP: Systolic blood pressure.

components of DNA and RNA, cell signaling, energy storage, transfer, and maintenance of acid-base equilibrium[16, 20, 22].

Decreased sPi concentration is a common phenomenon after non-traumatic ICH, including aSAH[17, 22], which may be caused by increased renal excretion, decreased intestinal absorption, or internal redistribution of inorganic phosphate[16, 22]. The redistribution of inorganic phosphate across cell membranes is the most common cause of hypophosphatemia in patients and can be caused by various clinical conditions[26]. The underlying mechanism is attributed to the following reasons. Firstly, spontaneous hyperventilation is very common in patients with aSAH[27]. In respiratory alkalosis, a decrease in carbon dioxide can trigger an increase in intracellular $\mathrm{pH}$, which stimulates phosphofructokinase, leading to increased glycolysis and the incorporation of phosphate into organic intermediates $[28,29]$. Therefore, this causes a decrease in phosphate in the cell, which transfers phosphate into the cell. Some reports in the literature have revealed that during hyperventilation, the patient's serum phosphate level is as low as $0.3 \mathrm{mmol} / \mathrm{L}[27]$, which may be the most common cause of hypophosphatemia in hospitalized patients. Reduced sPi concentration caused by respiratory alkalosis is also seen in patients with head injuries, acute strokes, and mechanical ventilation[28, 30]. Secondly, high serum levels of endogenous or exogenous catecholamines (such as epinephrine and norepinephrine) in patients after aSAH can lead to a decrease in serum phosphate[31]. Reported literature hinted sPi is inversely related to the paroxysmal sympathetic storm and increased plasma epinephrine[32]. Adrenaline can raise blood pressure, and our data also indicated that patients with lower SPi levels had higher SBP than those with higher sPi levels. Our findings are consistent with the literature in implying a negative correlation between the initial sPi concentration and plasma epinephrine[31, 33]. Thirdly, serum phosphate levels are negatively correlated with the levels of inflammatory cytokines such as interleukin 6 (IL-6) and tumor necrosis factor-alpha (TNF-a)[10, 34]. Elevated inflammatory factors lead to internal redistribution of phosphorus, and decreased serum phosphate may be due to increased use of phosphate by immune cells.

aHCP is not an uncommon complication and is a potentially treatable cause of early neurological deterioration following aSAH. Systemic inflammatory response syndrome is common in aSAH[12, 35]. aHCP is characterized by enlargement of the cerebrospinal fluid (CSF)-filled brain ventricles from failed CSF homeostasis. Since the 1840's, inflammation in the brain and the CSF spaces have been detected in both post-hemorrhagic and post-infectious hydrocephalus[36]. Inflammatory cells might be an independent predictive factor associated with aHCP after aSAH. Recent studies have begun to reveal the 
Characteristics

Lower serum

Phosphorus

( $\leq 1.00 \mathrm{mmol} / \mathrm{L})$

312

84

$54.67 \pm 10.38$

186(59.6)

54(17.3)

36(11.5)

157(50.3)

33(10.6)

48(15.4)

$145.23 \pm 26.74$

$84.61 \pm 14.78$

DBP, $\mathrm{mmHg}$

Hunt-Hess grade ( $N$, \%)

Grade $\mathbb{X}-\mathbb{\square}$

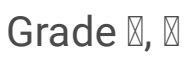

mFisher grade ( $N$, \%)

Grade 0-2

Grade 3-4

Aneurysm characteristics ( $N$,

\%)

Multiple aneurysms

Anterior circulation

aneurysm

Time from onset to admission,h

66(21.2)

166(53.2)

45(14.4)

265(84.9)

$14.0(8.0-18.0)$
Higher serum

Phosphorus

$(\nabla 1.00 \mathrm{mmol} / \mathrm{L})$

323

39

$\bigotimes 0.001$

$53.86 \pm 11.13$

0.342

214(66.3)

0.083

0.465

49(15.2)

0.426

31(9.6)

0.227

178(55.1)

0.882

33(10.2)

0.285

60(18.6)

0.001

$138.79 \pm 13.55$

0.709

$84.20 \pm 13.29$

$\varangle 0.001$

293(90.7)

$30(9.3)$

$\llbracket 0.001$

230(71.2)

93(28.8)

0.438

37(11.5)

285(88.2)

15.0(10.0-17.0)

0.949

\section{Admission laboratory}




\begin{tabular}{|c|c|c|c|}
\hline Serum potassium, $\mathrm{mmol} / \mathrm{L}$ & $3.83(3.56-4.10)$ & $4.05(3.74-4.33)$ & 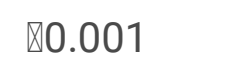 \\
\hline Serum sodium, $\mathrm{mmol} / \mathrm{L}$ & $140.45(137.60-142.80)$ & $141.0(139.0-143)$ & 0.038 \\
\hline Serum calcium, mmol/L & $2.13(2.04-2.21)$ & $2.22(2.14-2.30)$ & $\nabla 0.001$ \\
\hline \multicolumn{3}{|l|}{ Treatment $(\mathrm{N}, \%)$} & 0.053 \\
\hline Clipping & $226(72.4)$ & $211(65.3)$ & \\
\hline Coiling & $86(27.6)$ & 112(34.7) & \\
\hline \multicolumn{3}{|l|}{ mRS (N, \%) } & 0.015 \\
\hline $0-2$ & 259(83) & $290(89.8)$ & \\
\hline $3-6$ & $53(17)$ & $33(10.2)$ & \\
\hline \multicolumn{4}{|c|}{$\begin{array}{l}\text { aSAH: aneurysmal subarachnoid hemorrhage; aHCP: acute hydrocephalus; CI: Confidence interval; } \\
\text { DBP: Diastolic blood pressure; IQR: Interquartile range; mFisher: modified Fisher; OR: Odds ratios; SPi } \\
\text { serum Phosphate ion; SD: Standard deviation; SBP: Systolic blood pressure. }\end{array}$} \\
\hline
\end{tabular}

molecular mechanisms by which inflammation-regulating cytokines, immune cells, and signaling pathways- contribute to the pathogenesis of hydrocephalus[10,36]. The cytokines, immune cells, and signal pathways all require the participation of phosphate. aSAH can accelerate cell apoptosis. Phosphate is released from dead cells into blood vessels, promotes vascular inflammation and endothelial cell apoptosis[36]. In the present study, a reduction of serum phosphate was observed in patients with aHCP following aSAH, supporting a possible link between phosphate metabolism, inflammation, and hydrocephalus.

Numerous mechanisms have been implicated as causative factors for the development of aHCP following aSAH, including obstruction of the arachnoid granulations by blood products, alterations in CSF dynamics, and adhesions within the ventricular system[6, 10]. Emerging data have established that inflammatory pathways are likely to play an essential role in the pathogenesis of aHCP. Inflammatory markers in CSF and peripheral blood are associated with the possibility of developing aHCP $[9,10,12,36]$. Lower sPi levels are negatively correlated with the levels of inflammatory cytokines[16]. This study determined that a lower admission sPi level is considered a risk factor for aHCP. It seems consistent with previous literature that Inflammation can induce aHCP[36].

Impaired early energy metabolism in the brain may be another mechanism that causes aHCP after aSAH. In this study, we observed decreased serum phosphate was associated with higher severity of aSAH, consistent with existing literature [17]. Phosphate is the most abundant intracellular anion and is involved in synthesizing various enzymes in the body. It is necessary to produce ATP and 2,3-diphosphoglycerate in red blood cells, which helps to release oxygen from hemoglobin[37]. Therefore, a decreased sPi level is likely to shift the hemoglobin dissociation curve to the right and increase the affinity of hemoglobin for oxygen, resulting in a decrease in oxygen release, thereby impairing brain energy metabolism, aggravating brain damage, and affecting the virtuous circulation of CSF[38]. The above process may lead 
to subsequent aHCP. In addition, lower sPi levels may aggravate the destruction of the blood-brain barrier and further aggravate the occurrence of aHCP.

The influence of sPi levels on the prognosis of critically ill patients, including aSAH, is controversial[17, 22]. Our current data demonstrated that lower sPi levels at admission were observed in patients with unfavorable outcomes. However, according to the ROC curve, the AUC of sPi is 0.602 for mRS, indicating that the predictive value of $\mathrm{sPi}$ in predicting poor prognosis is weak. The presence of a lower sPi level may be just another indicator of disease severity, not a major contributor factor to disease severity. This hypothesis is supported by our findings that there is a negative correlation between the initial phosphate concentration and the commonly used disease severity scores, including mFisher score and Hunt-Hess grade. This association has been observed in some previous studies, but the opposite results have also been reported[17]. Of course, their research objects are not patients with sICH. Therefore, it seems reasonable that the conclusions are different.

\section{Limitation}

Limitations of this study include sample size and observational single-center study, which may limit the generality of our results. Furthermore, all laboratory data are initial values at admission, and increased dynamic analysis of follow-up data may help improve our findings. Thirdly, factors affecting serum phosphorus have not been considered, including eating habits, food intake, and hormone levels. Finally, related inflammatory cytokines, such as $C$ reactive protein, procalcitonin, and IL-6, were not included in the analysis

\section{Conclusion}

Lower serum phosphorus levels predict the occurrence of aHCP, and the model constructed by sPi levels, Hunt-Hess grade, and mFisher scale significantly improves the prediction of aHCP after aSAH. Further multicenter randomized studies are needed to determine whether changes in sPi levels over time are related to the occurrence of aHCP.

\section{Abbreviations}

aSAH: aneurysmal subarachnoid hemorrhage; aHCP: acute hydrocephalus; $\mathrm{AUC}=$ area under the curve; $\mathrm{Cl}$ : Confidence interval; CSF: Cerebrospinal fluid; CT: computed tomography; CTA: Computerized tomography angiography; DBP: Diastolic blood pressure; DSA: Digital subtraction angiography; ICH: Intracranial hemorrhage; ICU: Intensive care unit; IL-6:Interleukin-6; IQR: Interquartile range; mRS: modified Rankin Scale; mFisher: modified Fisher; OR: Odds ratios; PSM: Propensity-score matching; ROC: Receiver operating curve; sPi: serum Phosphate ion; SD: Standard deviation; SBP: Systolic blood pressure; TNFa:tumor necrosis factor-alpha. 


\section{Declarations}

\section{Acknowledgments}

Not applicable.

\section{Authors' contributions}

YBZ, SFZ designed the study, collected and analyzed data, and drafted the manuscript; GRC helped in the statistical analysis and result interpretation; GRC, CWL, PSY prepared the figures and interpret the results; YXL supervised study; PSY and DZK were identified as the guarantor of the paper, taking responsibility for the integrity of the work as a whole. All authors read and approved the final manuscript.

\section{Sources of Funding}

Not applicable.

\section{Availability of data and materials}

The datasets generated and/ or analyzed during the current study are available from the corresponding author upon reasonable request.

\section{Ethics approval and consent to participate}

The authors are accountable for all aspects of the work in ensuring that questions related to the accuracy or integrity of any part of the work are appropriately investigated and resolved. This study utilized the anonymous data available in the database with pre-existing institutional review board approval.

\section{Consent for publication}

Not applicable.

\section{Competing interests}

The authors have no competing interests to declare.

\section{Author details}


1.Department of Neurosurgery, Neurosurgery Research Institute, the First Affiliated Hospital, Fujian Medical University, Fujian province, P. R. China.

2.Fujian Key Laboratory of Precision Medicine for Cancer, the First Affiliated Hospital, Fujian Medical University, Fujian province, P. R. China.

3.Key Laboratory of Radiation Biology of Fujian higher education institutions, the First Affiliated Hospital, Fujian Medical University, Fujian province, P. R. China.

4.Clinical research and translation center, the First Affiliated Hospital, Fujian Medical University, Fujian province, P. R. China.

Yi-Bin Zhang, Shu-Fa Zheng, and Guo-Rong Chen contributed equally to this article.

\section{References}

1. Lawton MT, Vates GE. Subarachnoid Hemorrhage. N Engl J Med. 2017;377(3): 257266.https://doi.org/10.1056/NEJMcp1605827.

2. Diringer MN. Management of aneurysmal subarachnoid hemorrhage. Crit Care Med. 2009;37(2): 432440.https://doi.org/10.1097/CCM.0b013e318195865a.

3. Macdonald RL, Schweizer TA. Spontaneous subarachnoid haemorrhage. The Lancet. 2017;389(10069): 655-666.https://doi.org/10.1016/s0140-6736(16)30668-7.

4. Diesing D, Wolf S, Sommerfeld J, Sarrafzadeh A, Vajkoczy P, Dengler NF. A novel score to predict shunt dependency after aneurysmal subarachnoid hemorrhage. J Neurosurg. 2018;128(5): 12731279.https://doi.org/10.3171/2016.12.JNS162400.

5. Jovanovic I, Nemir J, Gardijan D, et al. Transient acute hydrocephalus after aneurysmal subarachnoid hemorrhage and aneurysm embolization: a single-center experience. Neuroradiology. 2021.https://doi.org/10.1007/s00234-021-02747-2.

6. Mehta V, Holness RO, Connolly K, Walling S, Hall R. Acute Hydrocephalus Following Aneurysmal Subarachnoid Hemorrhage. The Canadian journal of neurological sciences. 1996;23(1): 40-45.

7. Connolly ES, Jr., Rabinstein AA, Carhuapoma JR, et al. Guidelines for the management of aneurysmal subarachnoid hemorrhage: a guideline for healthcare professionals from the American Heart Association/american Stroke Association. Stroke. 2012;43(6): 1711-

1737.https://doi.org/10.1161/STR.0b013e3182587839.

8. Jartti P, Karttunen A, Jartti A, Ukkola V, Sajanti J, Pyhtinen J. Factors related to acute hydrocephalus after subarachnoid hemorrhage. Acta Radiol. 2004;45(3): 333339.https://doi.org/10.1080/02841850410004274.

9. Kuo LT, Huang AP. The Pathogenesis of Hydrocephalus Following Aneurysmal Subarachnoid Hemorrhage. Int J Mol Sci. 2021;22(9).https://doi.org/10.3390/ijms22095050. 
10. Chen S, Luo J, Reis C, Manaenko A, Zhang J. Hydrocephalus after Subarachnoid Hemorrhage: Pathophysiology, Diagnosis, and Treatment. Biomed Res Int. 2017;2017: 8584753.https://doi.org/10.1155/2017/8584753.

11. Demirgil BT, Tugcu B, Postalci L, Guclu G, Dalgic A, Oral Z. Factors leading to hydrocephalus after aneurysmal subarachnoid hemorrhage. Minim Invasive Neurosurg. 2003;46(6): 344348.https://doi.org/10.1055/s-2003-812500.

12. Hokari M, Uchida K, Shimbo D, Gekka M, Asaoka K, Itamoto K. Acute systematic inflammatory response syndrome and serum biomarkers predict outcomes after subarachnoid hemorrhage. $J$ Clin Neurosci. 2020;78: 108-113.https://doi.org/10.1016/j.jocn.2020.05.055.

13. Wilson CD, Safavi-Abbasi S, Sun H, et al. Meta-analysis and systematic review of risk factors for shunt dependency after aneurysmal subarachnoid hemorrhage. J Neurosurg. 2017;126(2): 586595.https://doi.org/10.3171/2015.11.JNS152094.

14. Loggini A, El Ammar F, Mansour A, Kramer CL, Goldenberg FD, Lazaridis C. Association between electrolyte levels at presentation and hematoma expansion and outcome in spontaneous intracerebral hemorrhage: A systematic review. J Crit Care. 2021;61: 177185.https://doi.org/10.1016/j.jcrc.2020.10.029.

15. Leung J, Crook M. Disorders of phosphate metabolism. J Clin Pathol. 2019;72(11): 741747.https://doi.org/10.1136/jclinpath-2018-205130.

16. Florenzano P, Cipriani C, Roszko KL, et al. Approach to patients with hypophosphataemia. The Lancet Diabetes \& Endocrinology. 2020;8(2): 163-174.https://doi.org/10.1016/s2213-8587(19)30426-7.

17. Junttila E, Koskenkari J, Ala-Kokko T. Hypophosphatemia after nontraumatic intracranial hemorrhage. Acta Anaesthesio/ Scand. 2017;61(6): 641-649.https://doi.org/10.1111/aas.12903.

18. U.Weber, T.Hüppe, L.Niehaus. CT and MRI in severe hypophosphataemia with central nervous system involvement. Neuroradiology. 2000;42(2): 112-114.https://doi.org/10.1007/s002340050026.

19. Monsalve-Naharro, Á;Gerónimo-Pardo J, M;Campos-García ÖJ. Hypophosphataemia complicating the neurological picture of a patient with intracranial haemorrhage. Anaesthesia and Intensive Care. 2012;40(6): 1069-1070.

20. Kin P, JONES JD, THORALF M. SUNDT J. High-energy phosphate levels in the cerebral artery during chronic vasospasm after subarachnoid hemorrhage. Journal of Neurosurgery. 1996;76(6): 991996.https://doi.org/10.3171/jns.1992.76.6.0991.

21. Park W, Kim BS, Lee JE, et al. Serum phosphate levels and the risk of cardiovascular disease and metabolic syndrome: a double-edged sword. Diabetes Res Clin Pract. 2009;83(1): 119125.https://doi.org/10.1016/j.diabres.2008.08.018.

22. Geerse DA, Bindels AJ, Kuiper MA, Roos AN, Spronk PE, Schultz MJ. Treatment of hypophosphatemia in the intensive care unit: a review. Critical Care. 2010;14(4): R147.https://doi.org/10.1186/cc9215. Epub 2010 August 3.

23. Zhu Y, Jiang H, Li Y, et al. Serum Alkaline Phosphatase Level is Associated with Angiographic Vasospasm, Delayed Cerebral Ischemia-Caused Clinical Deterioration, and Functional Outcome After 
Aneurysmal Subarachnoid Hemorrhage. Neurocrit Care. 2019;31(3): 466475.https://doi.org/10.1007/s12028-019-00714-7.

24. Saver JL, Filip B, Hamilton S, et al. Improving the reliability of stroke disability grading in clinical trials and clinical practice: the Rankin Focused Assessment (RFA). Stroke. 2010;41(5): 992995.https://doi.org/10.1161/STROKEAHA.109.571364.

25. Yang $P$, Zhang Y, Zhang L, et al. Endovascular Thrombectomy with or without Intravenous Alteplase in Acute Stroke. N Engl J Med. 2020;382(21): 1981-1993.https://doi.org/10.1056/NEJMoa2001123.

26. Ziegler TR. Parenteral Nutrition in the Critically III Patient. The new england journal of medicine. 2009;361(11): 1088-1097.https://doi.org/10.1056/NEJMct0806956.

27. Williamson CA, Sheehan KM, Tipirneni R, et al. The Association Between Spontaneous Hyperventilation, Delayed Cerebral Ischemia, and Poor Neurological Outcome in Patients with Subarachnoid Hemorrhage. Neurocrit Care. 2015;23(3): 330-338.https://doi.org/10.1007/s12028015-0138-5.

28. Paleologos M, Stone E, Braude S. Persistent, progressive hypophosphataemia after voluntary hyperventilation. Clinical Science. 2000;98(5): 619-625.

29. Zhang D, Zhuang Z, Wei Y, et al. Association of Admission Serum Glucose-Phosphate Ratio with Severity and Prognosis of Aneurysmal Subarachnoid Hemorrhage. World Neurosurg. 2019;127: e1145-e1151.https://doi.org/10.1016/j.wneu.2019.04.071.

30. Valimaki V, Luostarinen T, Satopaa J, Raj R, Virta JJ. Neurointensive care results and risk factors for unfavorable outcome in aneurysmatic SAH: a comparison of two age groups. Acta Neurochir (Wien). 2021;163(5): 1469-1478.https://doi.org/10.1007/s00701-021-04731-4.

31. Body JJ, Cryer PE, Offord KP, 3rd HH. Epinephrine is a Hypophosphatemic Hormone in Man. The journal of clinical investigation. 1983;71(3): 572-578.https://doi.org/10.1172/jci110802.

32. Ko SB, Kim CK, Lee SH, Bae HJ, Yoon BW. Morphine-sensitive paroxysmal sympathetic storm in pontine intracerebral hemorrhage. Neurologist. 2010;16(6): 384-

385.https://doi.org/10.1097/NRL.0b013e3181b1cdff.

33. Wang X, Pei J, Hu X. The Brain-Heart Connection in Takotsubo Syndrome: The Central Nervous System, Sympathetic Nervous System, and Catecholamine Overload. Cardiol Res Pract. 2020;2020: 4150291.https://doi.org/10.1155/2020/4150291.

34. Yoshimoto Y, Tanaka Y, Hoya K. Acute Systemic Inflammatory Response Syndrome in Subarachnoid Hemorrhage. Stroke. 2001;32(9): 19989-11193.https://doi.org/10.1161/hs0901.095646.

35. Zuo Y, Huang L, Enkhjargal B, et al. Activation of retinoid $X$ receptor by bexarotene attenuates neuroinflammation via PPARgamma/SIRT6/FoxO3a pathway after subarachnoid hemorrhage in rats. J Neuroinflammation. 2019;16(1): 47.https://doi.org/10.1186/s12974-019-1432-5.

36. Karimy JK, Reeves BC, Damisah E, et al. Inflammation in acquired hydrocephalus: pathogenic mechanisms and therapeutic targets. Nat Rev Neurol. 2020;16(5): 285296.https://doi.org/10.1038/s41582-020-0321-y. 
37. Larsen VH, T Waldau HG, Siggaard-Andersen O. Erythrocyte 2,3-diphosphoglycerate depletion associated with hypophosphatemia detected by routine arterial blood gas analysis. Scand J Clin Lab Invest. 1996;56: 83-87. https://doi.org/10.3109/00365519609088626.

38. Macdonald RL. Delayed neurological deterioration after subarachnoid haemorrhage. Nat Rev Neurol. 2014;10(1): 44-58. https://doi.org/10.1038/nrneurol.2013.246.

\section{Figures}


1312 aSAH patients

17 patients who were transferred to other hospitals

40 patients who gave up treatment

1255 aSAH patients hospitalized

- 7 patients $<19$ years

- 1 patients with familial hypophosphatemia

- 1 patients parathyroid disease

- 17 patients missing clinical demographic

- 9 patients with systemic comorbidities

- 12 patients with brain tumor

- 15 patients with trauma

- 14 patients with acute kidney injury or chronic renal disease

- 13 patients with Mayomoya disease

- 23 patients with neurological disease

- 10 patients with postoperative hydrocephalus

- 472 patients who underwent emergency aneurysm clipping or endovascular coiling within 72 hours of aSAH

- 26 patients who were follow-up loss

123 patients who were acute hydrocephalus

\section{Figure 1}

The flowchart of this study is present in Figure 1. 
A

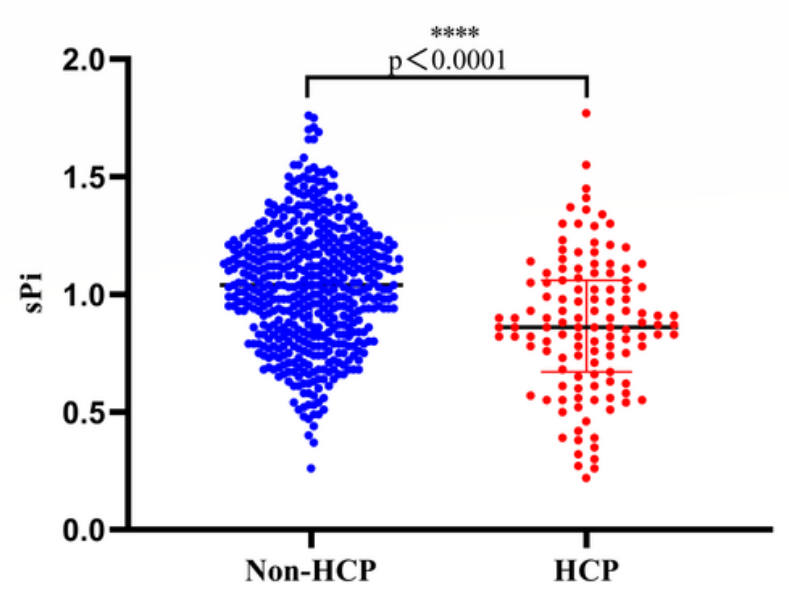

C

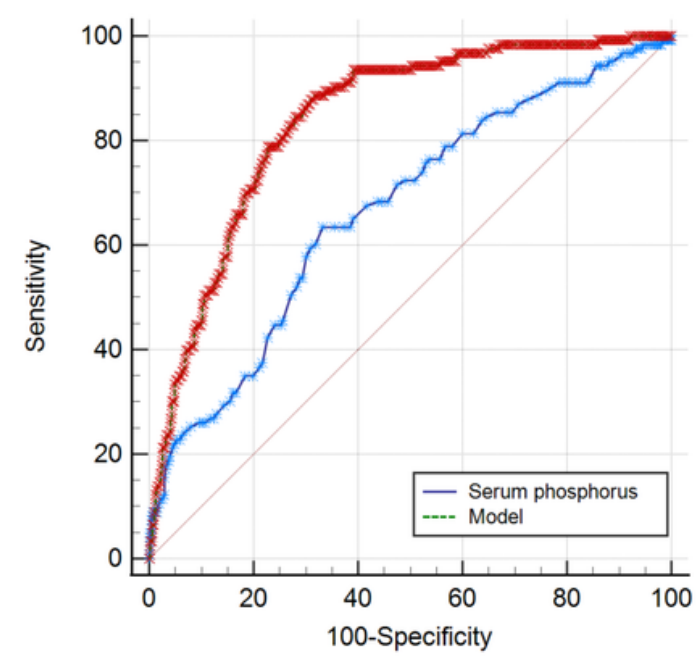

B

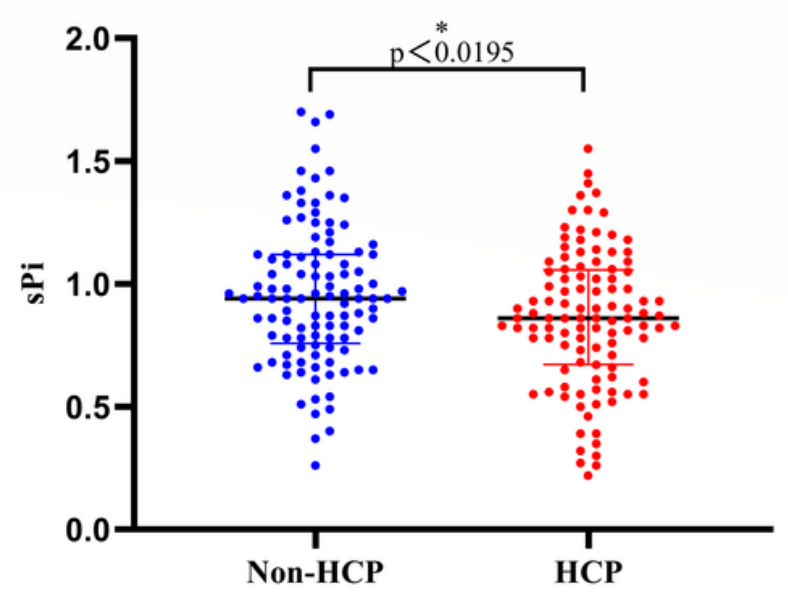

D

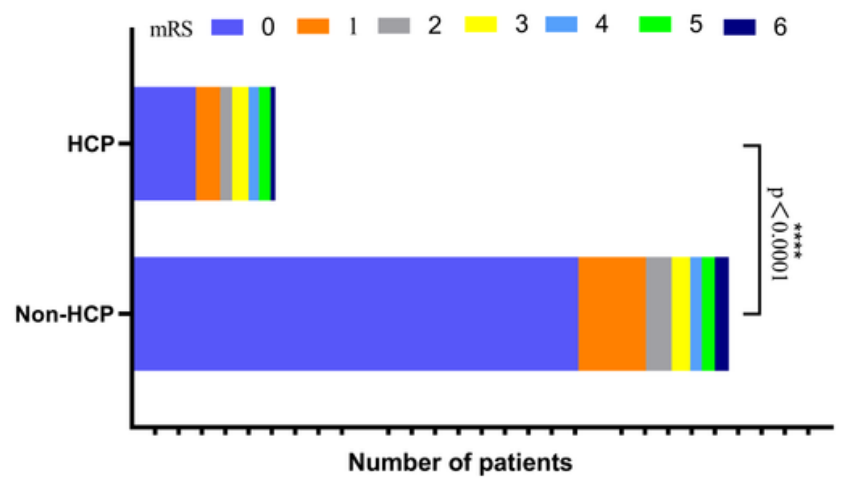

\section{Figure 2}

Lower sPi levels were detected in patients with aHCP compared with those without [0.86(0.67-1.06) $\mathrm{mmol} / \mathrm{L}$ vs. $1.04(0.84-1.21) \mathrm{mmol} / \mathrm{L}, \mathrm{P}<0.001]$. (Table1 and Fig.2A). Multivariable analysis revealed that lower sPi level, higher mFisher score, and higher Hunt-Hess grade were associated with aHCP (Table 1; sPi, OR 1.729, 95\% Cl 1.139-2.623, $\mathrm{P}=0.01$; mFisher OR 0.097,95\% Cl 0.055-0.172, $\mathrm{P}<0.001$; Hunt-Hess, OR $0.555,95 \% \mathrm{Cl} 0.320-0.961, \mathrm{P}=0.036$ ), while hypertension, diabetes mellitus, $S B P, D B P$, serum potassium and serum calcium were not associated with aHCP. After PSM, no significant differences were observed in hypertension, diabetes mellitus, SBP, DBP, serum potassium and serum calcium, admission Hunt-Hess grade, Fisher grade between HCP and non-HCP groups. The matched HCP group had a significantly lower sPi level than the matched non-HCP [(0.86(0.67-1.06) vs. 0.94(0.76-1.12)) mmol/L, $\mathrm{P}=0.020$; Fig. 2B]. ROC curve analysis shows an AUC (area under the curve) of 0.667 (95\% Cl 0.629-0.704, Pख0.0001) (Sensitivity=63.4\%; Specificity=66.8\%), and the best cutoff value for sPi level as a predictor for aHCP in aSAH patients was determined as $0.93 \mathrm{mmol} / \mathrm{L}$. The AUC of the logistic regression model based on these predictors (sPi, Hunt-Hess grade, and mFisher grade) was 0.840 ( $95 \% \mathrm{Cl} 0.809-0.868, \mathrm{P} \otimes 0.0001)$ with a 
sensitivity of $88.6 \%$ and specificity of $68.4 \%$ for aHCP, which is a stronger hydrocephalus predictor than a

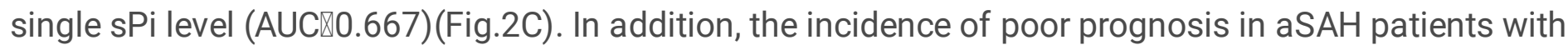
HCP was higher than those without HCP (Fig. 2D).
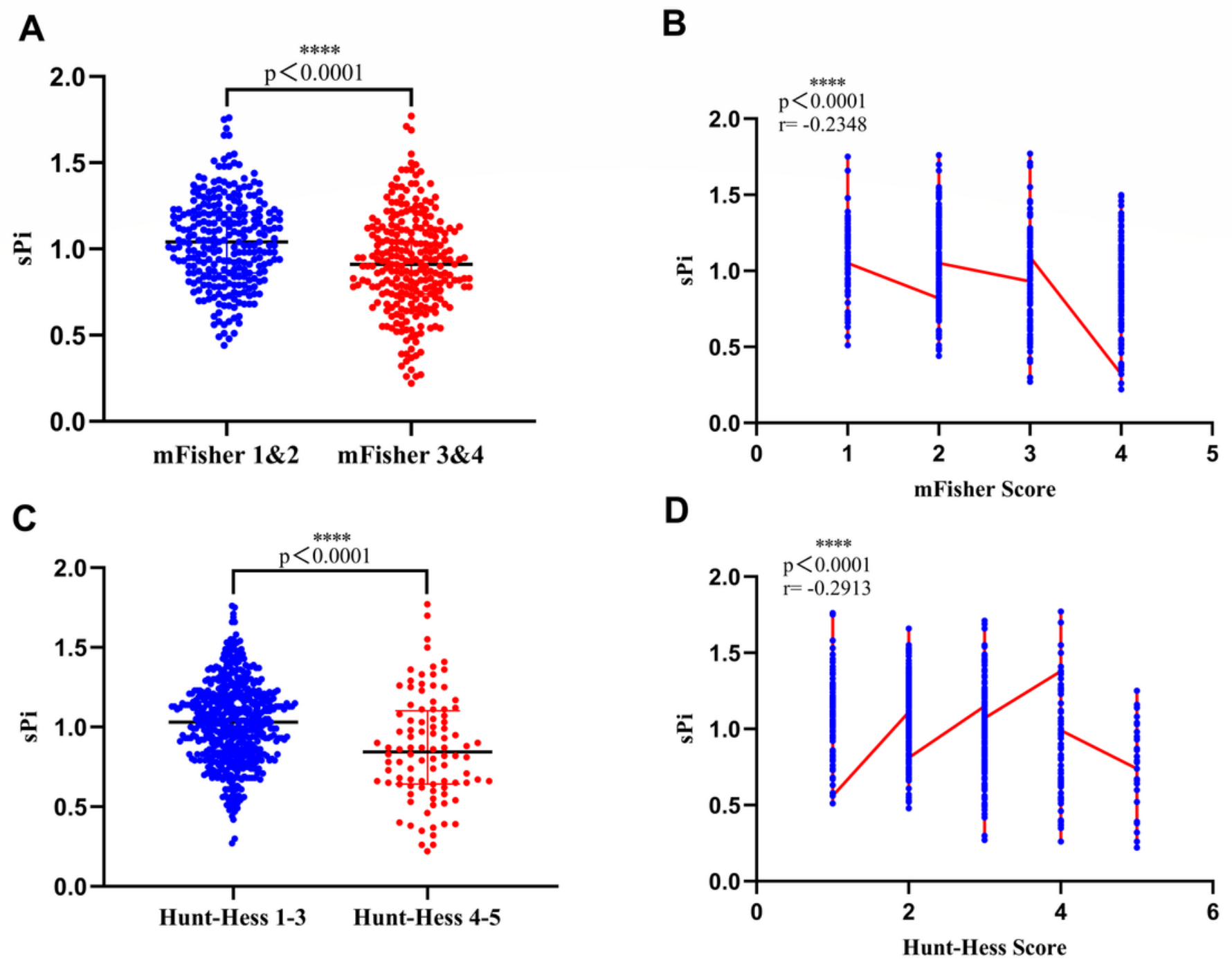

\section{Figure 3}

The sPi level was significantly lower in aSAH patients with $\mathrm{mFisher} \mathrm{grade} \mathrm{(3-4)} \mathrm{compared} \mathrm{to} \mathrm{mFisher} \mathrm{(1-}$

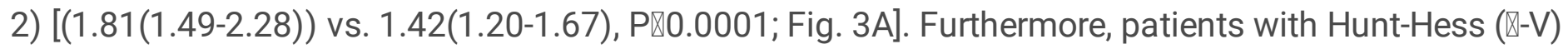

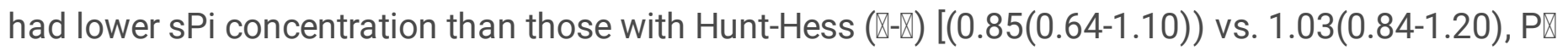
0.0001; Fig. 3B]. Spearman analyses revealed a clear negative correlation between sPi and mFisher score $(r=-0.2348, P<0.001$, Fig.3B), between $s P i$ and Hunt-Hess grade $(r=-0.2913, P<0.001$, Fig.3D), respectively. Correlation analyses revealed a negative correlation between sPi and SBP $(r=-0.137, P=0.001)$. No correlation was detected between sPi and age or sex(Pख0.05). 
A

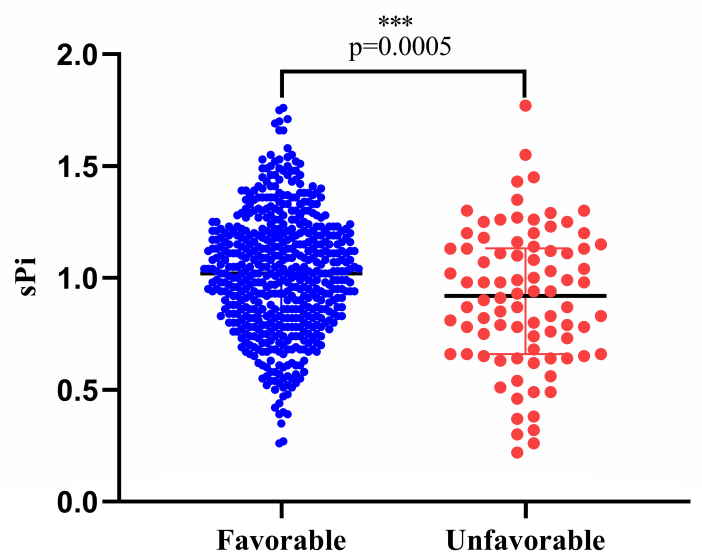

B

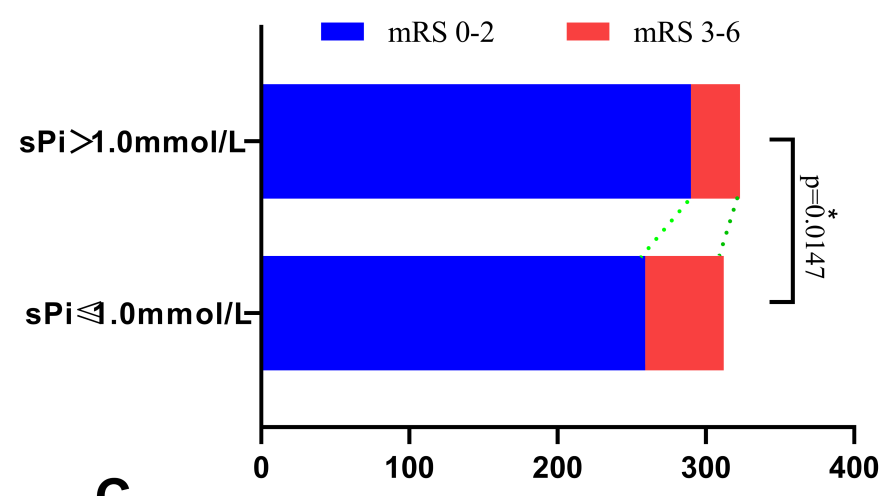

C

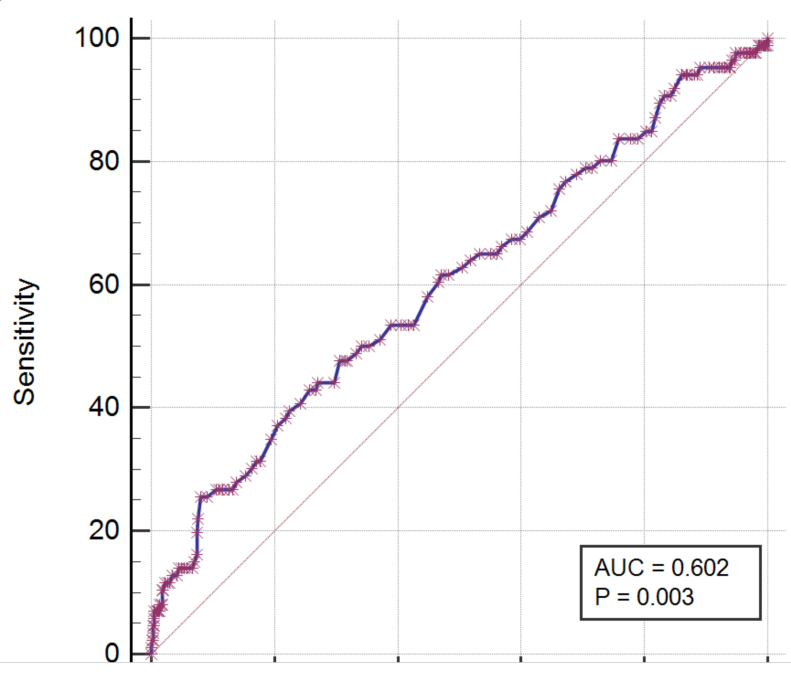

Figure 4

Compared with the favorable functional group, the sPi level at admission was markedly lower in the unfavorable functional group [0.92(0.66-1.13) vs. $1.02(0.83-1.20) \mathrm{mmol} / \mathrm{L}, \mathrm{P}=0.0005$, Fig.4A]. Patients with a sPi level below $1.0 \mathrm{mmol} / \mathrm{L}$ (the median level of $\mathrm{sPi}$ in all patients) have a more unfavorable outcome than patients with a sPi level $>1.0 \mathrm{mmol} / \mathrm{L}$ (Fig. 4B). According to the ROC curve, the AUC of sPi 
was $0.602(95 \% \mathrm{Cl} 0.563-0.640 ; \mathrm{P}=0.003)$ (Sensitivity=25.58\%; Specificity=91.99\%) for $\mathrm{mRS}$ based on a cut-off value of $0.66 \mathrm{mmol} / \mathrm{L}$ (Fig.4C). 